\title{
Un vent de changement
}

\author{
Patrick Fitch
}

\author{
Le changement est la loi de la vie. Et ceux dont le regard \\ est uniquement tourné vers le passé ou \\ le présent sont assurés de passer à côté de l'avenir. \\ - John F Kennedy
}

$\int \mathrm{e}$ suis en terrain connu. Certains d'entre vous s'en souviendront, j'ai autrefois occupé le poste de directeur des finances de la Société canadienne des pharmaciens d'hôpitaux (SCPH) de 2008 à 2012. Je suis maintenant de retour pour entamer un mandat de trois ans comme délégué présidentiel à partir du 22 octobre à Régina.

D'une certaine façon, c'est comme rentrer à la maison. J'ai grandi à Régina et j'y ai décroché mon premier emploi en pharmacie. La perspective de retourner dans la ville de mes jeunes années pour amorcer ce mandat à la direction m’a amené à réfléchir sur certains changements qui se sont opérés depuis que j'ai quitté le conseil de la SCPH il y a quelques années.

Le poste de directeur des finances se nomme maintenant trésorier. Le crépuscule des Séances éducatives d'été a eu lieu et 2016 marque la première année d'une nouvelle forme pour l'Assemblée générale annuelle de la Société. Cette réunion a été tenue le samedi 22 octobre conjointement avec la propre Assemblée générale annuelle et les séances éducatives 2016 de la section de la Saskatchewan.

La Conférence sur la pratique professionnelle fait peau neuve elle aussi. Elle aura notamment lieu au InterContinental Toronto Centre en 2017. Une chose qui restera inchangée est la grande qualité de la programmation prévue par le comité des services éducatifs.

Les programmes de Pharm. D. de premier cycle sont devenus la norme partout au pays, étant soit déjà en place soit approuvés pour mise en œuvre prochainement dans presque toutes les facultés de pharmacie. Ces programmes de Pharm. D., où l'accent sur la formation expérientielle est encore plus prononcé, sont idéalement conçus pour former les pharmaciens étudiants en vue d'une nouvelle époque stimulante où les champs de pratique sont élargis. Partout au Canada, des pharmaciens prescrivent des médicaments de façon indépendante ainsi que des examens de laboratoire et ils administrent par injection des vaccins ainsi que des médicaments, et ceci ne représente qu'une partie de leurs nouvelles activités.
La décision de la Cour suprême du Canada dans l'affaire Carter c. Canada et l'adoption du projet de loi C-14 ont rendu possible que les pharmaciens soient appelés à participer à des cas d'aide médicale à mourir ou à conseiller d'autres fournisseurs de soins de santé au sujet de leurs patients ayant demandé une telle aide. Cette situation est remplie d'inconnus pour notre profession et, à ce jour, l'on ne connaît pas les dangers ou les possibilités que peut engendrer ce nouveau domaine de pratique. Afin de guider les pharmaciens, la SCPH a récemment publié le document Aide médicale à mourir : Déclaration de principes (http://www.cshp.ca/ $\mathrm{dms} / \mathrm{dms} V$ iew/2_Physician-Assisted-Dying-Position-Statement\%28French\%29.pdf). Dans cette déclaration de principes, la $\mathrm{SCPH}$ se prononce en faveur : du respect des droits et de l'autonomie du patient; de la mise en place de protections en vertu de la loi fédérale pour tous les pharmaciens qui choisissent de participer aux activités liées à l'aide médicale à mourir; et du droit à l'objection de conscience de tout professionnel de la santé, du moment que la continuité des soins n'est pas réduite.

Finalement, les médias sociaux ont conquis le monde. À mes débuts au conseil de la SCPH, « blogue » était un nouveau terme. Aujourd'hui, on dirait que tout un chacun a un blogue, un compte Instagram, un fil Twitter, un profil LinkedIn ou dispose d'une autre plateforme pour y émettre ses opinions. Bien qu'elles soient beaucoup utilisées pour des futilités, ces technologies représentent aussi d'excellents moyens de communiquer avec nos pairs, des experts et nos patients. J'admets avoir trois comptes Twitter, dont un pour ma vie professionnelle. Je suis abonné aux fils de revues scientifiques, d'associations professionnelles et d'autres pharmaciens. Twitter est devenu ma principale source d'information sur la profession et j'espère pouvoir l'utiliser pour communiquer avec les membres de la SCPH pendant mon mandat à la direction. Alors, suivez-moi sur @ICU_Patrick et voyons ensemble ce que nous réservent les trois prochaines années.

[Traduction par l'éditeur]

Patrick Fitch, BSP, ACPR, est devenu président désigné et agent de liaison interne de la Société canadienne des pharmaciens d'hôpitaux lors de la réunion du conseil qui a suivi son élection à l'Assemblée générale annuelle en octobre 2016. 